\title{
Lagrangian analysis of 'trivial' symmetries in models of gravity
}

\author{
Debraj Roy ${ }^{1}$ \\ ${ }^{1}$ S. N. Bose National Centre for Basic Sciences, Block-JD, \\ Sector III, Salt Lake, Kolkata-700098, India. \\ E-mail: debraj@bose.res.in
}

\begin{abstract}
We study the differences between Poincaré and canonical hamiltonian symmetries in models of gravity through the corresponding Noether identities and show that they are equivalent modulo trivial gauge symmetries.
\end{abstract}

\section{Introduction}

Poincaré symmetry is a fundamental symmetry of nature and a gauge theory of the Poincaré group can be used to model theories of gravity. This Poincaré gauge theory (PGT) was developed by Utiyama [12, Kibble [9], Sciama [11] and later on by various authors [6]. PGT is built on a global manifold with local orthonormal frames glued to each spacetime point by frame fields or triads (in 3D). The triads $b^{i}{ }_{\mu}$ are used to translate between the global (index: Greek) and local (index: Latin) frames. To construct a gauge theory, connections $\omega^{i}{ }_{\mu}$ are introduced replacing partial derivatives by corresponding covariant derivatives. The corresponding field strengths give rise to the gravitational fields of curvature $R^{i}{ }_{\mu \nu}$ and torsion $T_{\mu \nu}^{i}$

$$
\begin{aligned}
R_{\mu \nu}^{i} & =\partial_{\mu} \omega^{i}{ }_{\nu}-\partial_{\mu} \omega^{i}{ }_{\nu}+\epsilon^{i}{ }_{j k} \omega^{j}{ }_{\mu} \omega^{k}{ }_{\nu} \\
T^{i}{ }_{\mu \nu} & =\nabla_{\mu} b^{i}{ }_{\nu}-\nabla_{\nu} b^{i}{ }_{\mu} .
\end{aligned}
$$

These fields can now be used to write actions describing gravity in Riemann-Cartan spacetime. Imposition of a condition on torsion through equations of motion (in vacuum) may lead one to a spacetime with only curvature and no torsion - the usual Einstein GR on Riemannian manifold.

As gauge theories of the Poincaré group, Poincaré symmetries are already inbuilt. A Dirac canonical analysis of symmetries on the other 
hand also yield a set of gauge symmetries for the same models. By a gauge symmetry here we mean any continuous symmetry of the basic fields that leave the action invariant. The total number of independent gauge symmetries are however limited by the number of independent, primary first class constraints [8]. So it transpires that there is a discrepancy with established results in the apparent off-shell in-equivalence between the Poincaré and canonical hamiltonian symmetries. Here we study and resolve this from a lagrangian point of view.

\section{Noether identities and trivial symmetries}

For specifics of discussion, we take up the Mielke-Baekler model [10] describing a cosmologically topological model of gravity with torsion. The action for the model is

$$
\begin{aligned}
S=\int \mathrm{d}^{3} \mathrm{x} \epsilon^{\mu \nu \rho}\left[a b^{i}{ }_{\mu} R_{i \nu \rho}\right. & -\frac{\Lambda}{3} \epsilon_{i j k} b^{i}{ }_{\mu} b^{j}{ }_{\nu} b_{\rho}^{k}+\alpha_{3}\left(\omega^{i}{ }_{\mu} \partial_{\nu} \omega_{i \rho}\right. \\
& \left.\left.+\frac{1}{3} \epsilon_{i j k} \omega^{i}{ }_{\mu} \omega^{j}{ }_{\nu} \omega^{k}{ }_{\rho}\right)+\frac{\alpha_{4}}{2} b^{i}{ }_{\mu} T_{i \nu \rho}\right]
\end{aligned}
$$

where the terms are the Einstein-Cartan term, cosmological term, ChernSimons term (in connection) and the torsion term respectively. The Euler derivatives corresponding to the independent canonical fields are:

$$
\begin{aligned}
\frac{\delta S}{\delta b^{i}{ }_{\mu}} & =\epsilon^{\mu \nu \rho}\left[a R_{i \nu \rho}+\alpha_{4} T_{i \nu \rho}-\Lambda \epsilon_{i j k} b_{\nu}^{j} b_{\rho}^{k}\right] \\
\frac{\delta S}{\delta \omega^{i}{ }_{\mu}} & =\epsilon^{\mu \nu \rho}\left[\alpha_{3} R_{i \nu \rho}+a T_{i \nu \rho}+\alpha_{4} \epsilon_{i j k} b_{\nu}^{j} b_{\rho}^{k}\right]
\end{aligned}
$$

The model independent Poincaré symmetries (subscript ' $P$ ') are $[\underline{5}$

$$
\begin{aligned}
\delta_{P} b_{\mu}^{i} & =-\epsilon_{j k}^{i} b_{\mu}^{j} \theta^{k}-\partial_{\mu} \xi^{\rho} b_{\rho}^{i}-\xi^{\rho} \partial_{\rho} b_{\mu}^{i} \\
\delta_{P} \omega^{i}{ }_{\mu} & =-\partial_{\mu} \theta^{i}-\epsilon_{j k}^{i} \omega_{\mu}^{j} \theta^{k}-\partial_{\mu} \xi^{\rho} \omega_{\rho}^{i}-\xi^{\rho} \partial_{\rho} \omega_{\mu}^{i} .
\end{aligned}
$$

while the canonical symmetries generated by the first-class gauge generator constructed through an off-shell algorithm [8, 2, 3, are [1]

$$
\begin{aligned}
\delta_{H} b^{i}{ }_{\mu} & =\nabla_{\mu} \varepsilon^{i}-p \epsilon^{i}{ }_{j k} b_{\mu}{ }_{\mu} \varepsilon^{k}+\epsilon^{i}{ }_{j k} b^{j}{ }_{\mu} \tau^{k} \\
\delta_{H} \omega^{i}{ }_{\mu} & =\nabla_{\mu} \tau^{i}-q \epsilon^{i}{ }_{j k} b^{j}{ }_{\mu} \varepsilon^{k} .
\end{aligned}
$$

An inspection of the two symmetries (5) and (6) reveal that the canonical symmetries are structurally dependent on the form of the action while the Poincaré symmetries are independent of particular action. Also, to compare the two symmetries, we have to first find a suitable mapping 
between the different sets of gauge parameters. To find this, we take recourse to the Noether identities corresponding to the symmetries [4.

A Noether identity corresponds to a each continuous gauge symmetry of an action, marked by an independent gauge parameter. Infact, the identity is a direct consequence of the invariance of the action. To see this, let us consider a generic gauge symmetry expressed as terms proportional to the gauge parameter $\left(\varepsilon^{\mu}\right)$ and its derivative

$$
\delta q_{i}=R_{i \mu} \varepsilon^{\mu}+\tilde{R}_{i \mu}^{\nu}\left(\partial_{\nu} \varepsilon^{\mu}\right) .
$$

The invariance of the action, step by step, leads to

$$
\begin{aligned}
\delta S & =\int \frac{\delta \mathcal{L}}{\delta q_{i}} \delta q_{i}=\int \frac{\delta \mathcal{L}}{\delta q_{i}}\left(R_{i \mu} \varepsilon^{\mu}+\tilde{R}_{i \mu}{ }^{\nu} \partial_{\nu} \varepsilon^{\mu}\right) \\
& =\int\left[\frac{\delta \mathcal{L}}{\delta q_{i}} R_{i \mu}-\partial_{\nu}\left(\frac{\delta \mathcal{L}}{\delta q_{i}} \tilde{R}_{i \mu}{ }^{\nu}\right)\right] \varepsilon^{\mu}=0,
\end{aligned}
$$

where the quantity within braces form the Noether identity due to the arbitrary nature of each of the gauge parameters.

The Noether identities corresponding to PGT symmetries are

$$
\begin{aligned}
P_{k} & =\frac{\delta S}{\delta b^{i}{ }_{\mu}}{ }_{j k}{ }_{j k} b_{\mu}+\frac{\delta S}{\delta \omega_{\mu}^{i}} \epsilon_{j k}^{i} \omega_{\mu}^{j}-\partial_{\mu}\left(\frac{\delta S}{\delta \omega^{k}}\right)=0 \\
R_{\rho} & =\frac{\delta S}{\delta b^{i}{ }_{\mu}} \partial_{\rho} b^{i}{ }_{\mu}+\frac{\delta S}{\delta \omega^{i}{ }_{\mu}} \partial_{\rho} \omega^{i}{ }_{\mu}-\partial_{\mu}\left(b^{i}{ }_{\rho} \frac{\delta S}{\delta b^{i}{ }_{\mu}}+\omega^{i}{ }_{\rho} \frac{\delta S}{\delta \omega^{i}{ }_{\mu}}\right)=0
\end{aligned}
$$

and that corresponding to canonical hamiltonian symmetries of the MielkeBaekler action are

$$
\begin{aligned}
& A_{k}=\frac{\delta S}{\delta b^{i}{ }_{\mu}} \epsilon_{j k}^{i} b_{\mu}^{j}+\frac{\delta S}{\delta \omega^{i}{ }_{\mu}} \epsilon_{j k}^{i} \omega_{\mu}^{j}-\partial_{\mu}\left(\frac{\delta S}{\delta \omega^{k}{ }_{\mu}}\right)=0 \\
& B_{k}=-\partial_{\mu}\left(\frac{\delta S}{\delta b^{k}}\right)+\frac{\delta S}{\delta b^{i}{ }_{\mu}} \epsilon_{j k}^{i} \omega^{j}{ }_{\mu}-p \frac{\delta S}{\delta b^{i}{ }_{\mu}{ }^{i}{ }_{j k} b^{j}{ }_{\mu}-q \frac{\delta S}{\delta \omega^{i}{ }_{\mu}} \epsilon_{j k}^{i} b_{\mu}^{j}=0}
\end{aligned}
$$

A comparison between (9) and (10) immediately shows that one identity from each pair is already equivalent: $P_{k}=A_{k}$. Comparing the nature of the other identities it is seen that the term $-\omega^{k}{ }_{\rho} A_{k}-b_{\rho}^{k} B_{k}$ gives

$$
\begin{aligned}
-R_{\rho} & +\frac{\delta S}{\delta b^{i}{ }_{\mu}}\left(\frac{\alpha_{3}}{\triangle} \eta^{i j} \epsilon_{\mu \nu \rho}\right) \frac{\delta S}{\delta b^{j}{ }_{\nu}}+\frac{\delta S}{\delta b^{i}{ }_{\mu}}\left(\frac{-a}{\triangle} \eta^{i j} \epsilon_{\mu \nu \rho}\right) \frac{\delta S}{\delta \omega^{j}{ }_{\nu}} \\
& +\frac{\delta S}{\delta \omega^{i}{ }_{\mu}}\left(\frac{-a}{\triangle} \eta^{i j} \epsilon_{\mu \nu \rho}\right) \frac{\delta S}{\delta b^{j}{ }_{\nu}}+\frac{\delta S}{\delta \omega^{i}{ }_{\mu}}\left(\frac{\alpha_{4}}{\triangle} \eta^{i j} \epsilon_{\mu \nu \rho}\right) \frac{\delta S}{\delta b^{j}{ }_{\nu}}=0
\end{aligned}
$$

where $\triangle=2\left(\alpha_{3} \alpha_{4}-a^{2}\right)$. The terms proportional to square of Euler derivatives are antisymmetric in their co-efficients and as such drop out 
without having to use the equations of motion, i.e. without having to set the Euler derivatives to zero. Thus we get back the Poincaré Noether identities from the canonical hamiltonian Noether identities, their difference being just 'trivial' gauge identities [7. Thus substituting $R_{\rho}=-b_{\rho}^{k} B_{k}-\omega^{k}{ }_{\rho} A_{k}$ and $P_{k}=-A_{k}$ in $\delta S=\int\left(\theta^{k} P_{k}+\xi^{\rho} R_{\rho}\right)=0$ gives $\int\left[\left(-\theta^{k}-\xi^{\rho} \omega^{k}{ }_{\rho}\right) A_{k}+\left(-b^{k}{ }_{\rho} \xi^{\rho}\right) B_{k}\right]=0$. Comparing this with $\delta S=\int\left(\varepsilon^{k} A_{k}+\tau^{k} B_{k}\right)=0$ gives us the required map between the two sets of gauge parameters.

$$
\varepsilon^{i}=-\xi^{\rho} b_{\rho}^{i} \quad \& \quad \tau^{i}=-\theta^{i}-\xi^{\rho} \omega_{\rho}^{i} .
$$

So the Noether identities help us to generate the required map between different sets of gauge parameters and show the equivalence of the two symmetries as their difference is just 'trivial!'

\section{References}

[1] Banerjee, R., Gangopadhyay, S., Mukherjee, P. and Roy, D., "Symmetries of topological gravity with torsion in the hamiltonian and lagrangian formalisms", JHEP, 1002, 075, (2010). [DOI, 0912.1472.

[2] Banerjee, R., Rothe, H.J. and Rothe, K.D., "Hamiltonian approach to Lagrangian gauge symmetries", Phys. Lett. B, B463, 248-251, (1999). [DOI, hep-th/99060724.

[3] Banerjee, R., Rothe, H.J. and Rothe, K.D., "Master equation for Lagrangian gauge symmetries", Phys. Lett. B, B479, 429-434, (2000). [DOI], hep-th/9907217].

[4] Banerjee, R. and Roy, D., "Poincare gauge symmetries, hamiltonian symmetries and trivial gauge transformations", Phys. Rev. D, D84, 124034, (2011). [DOI], [1110.1720].

[5] Blagojevic, M. and Cvetkovic, B., "Canonical structure of 3-D gravity with torsion", Trends in General Relativty and Quantum Cosmology, 2, pp. 85-107, (Nova Science Publishers, New York, 2006). gr-qc/0412134.

[6] Hehl, F.W., von der Heyde, P., Kerlick, G.D. and Nester, J.M., "General relativity with spin and torsion: Foundation and prospects", Rev. Mod. Phys., 48, 393-416, (1976).

[7] Henneaux, M. and Teitelboim, C., Quantization of Gauge Systems, (Princeton University Press, Princeton, NJ, 1992). Google Books.

[8] Henneaux, M., Teitelboim, C. and Zanelli, J., "Gauge invariance and degree of freedom count", Nucl. Phys. B, B332, 169-188, (1990). [DOI].

[9] Kibble, T.W.B., "Lorentz invariance and the gravitational field", J. Math. Phys., 2, 212-221, (1961). DOI.

[10] Mielke, E. W. and Baekler, P., "Topological gauge model of gravity with torsion", Phys. Lett. A, A156, 399-403, (1991). [DOI].

[11] Sciama, D.W., in Recent Developments in General Relativity, Festschrift for Infeld, (Pergamon, New York, U.S.A., 1962).

[12] Utiyama, R., "Invariant theoretical interpretation of interactions", Phys. Rev., 101, 1597-1607, (1956). [DOI]. 